\title{
Bias Varies for Bioimpedance Analysis and Skinfold Technique when Stratifying Collegiate Male Athletes Fat-free Mass Hydration Levels
}

\author{
MISAEL DUQUE1, RONALD L. SNARR2, GREG A. RYAN2, KYUNG-SHIN PARK1,
} BRETT S. NICKERSON1

${ }^{1}$ Exercise Physiology Laboratory; College of Nursing and Health Sciences; Texas A\&M International University; Laredo, TX

2Department of Health Sciences \& Kinesiology; Georgia Southern University; Statesboro, GA

\section{Category: Undergraduate}

Advisor / Mentor: Nickerson, Brett (brett.nickerson@tamiu.edu)

\section{ABSTRACT}

Bioelectrical impedance analysis (BIA) and skinfold (SF) techniques are commonly used to estimate body composition in athletic settings. Both methods are based upon a 2-compartment (2C) model approach, which assumes the hydration of fat-free mass (FFM) is constant $(73.80 \%)$. Deviations from assumed constants such as FFM hydration have previously been observed in athletes. However, the magnitude of error associated with deviations in FFM hydration are scarce. PURPOSE: The purpose of this study was to evaluate the accuracy of BIA- and SF-based body fat percentage (BF\%) estimates in collegiate athletes when stratifying FFM hydration levels. METHODS: FFM hydration levels for the entire sample ranged from $64.55-73.84 \%$. Therefore, athletes were analyzed as a whole (FFM-Hydration $\left.\mathrm{ALL}_{\mathrm{L}} \mathrm{n}=63\right)$ and at the FFM hydration levels of $64.00-68.99 \%$ (FFM-Hydration $\mathrm{L}_{1}: \mathrm{n}=37$ ) and $69.00-74.00 \%$ (FFM-Hydration $\mathrm{L}_{\mathrm{L} 2}$ : $\mathrm{n}=26$ ). A 3-compartment model utilizing air displacement plethysmography for body volume and bioimpedance spectroscopy for total body water was employed in order to determine the accuracy of BIA and SF for the 3 separate groups. RESULTS: The results of this study demonstrated that BIA had significant constant error (CE) when analyzed in FFM-Hydration ${ }_{\mathrm{ALL}}$, FFM-Hydration ${ }_{\mathrm{L} 1}$, and FFMHydration $_{\mathrm{L} 2}$ (all $\mathrm{p}<0.001 ; \mathrm{CE}=5.64,6.27$, and $4.73 \%$, respectively). However, the $\mathrm{CE}$ was not statistically significant for SF when evaluating FFM-Hydration ${ }_{\mathrm{ALL}}, \mathrm{FFM-Hydration} \mathrm{L}_{\mathrm{L} 1}$ and FFM-Hydration $\mathrm{L}_{22}$ (all $\mathrm{p}>$ $0.05 ; \mathrm{CE}=-0.04,-1.36$, and $1.83 \%$, respectively). The BIA device revealed proportional bias for FFMHydration $_{\mathrm{ALL}}$ and FFM-Hyrdration 1 (coefficients $=-0.19$ and -0.21 ; both $\mathrm{p}<0.05$ ). However, the proportional bias was not present for BIA when analyzed in FFM-Hydration L2 $_{\text {(coefficient }}=-0.16 ; \mathrm{p}=$ 0.06). Lastly, the SF method only had significant proportional bias when examined in FFM-Hydration $A L L$ (coefficient $=0.17 ; p=0.02$ ). CONCLUSIONS: The current study results revealed that proportional bias for BIA is removed when FFM hydration levels approach the assumed $73.80 \%$ commonly employed in $2 \mathrm{C}$ models. In contrast, the large variance in FFM hydration levels did not impact the SF technique. Therefore, practitioners are encouraged to utilize the $\mathrm{SF}$ technique over BIA when assessing $\mathrm{BF} \%$ in collegiate male athletes. 